\title{
النقل الضبابي المقيد متعدد الاهداف مع قيود مختلطتّ باستخدام دوال انتماء مختلفتش
}

م.م. علاء شنيشل جيتر/ الجامعت المستنصريت / كليتت التوبيت البدنيت وعلوم الرياضت

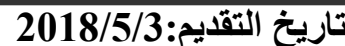

تاريخ القبول:2018/6/13

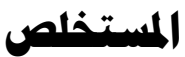

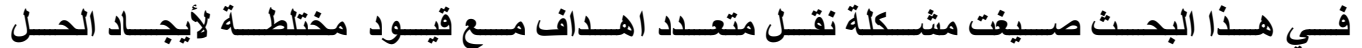

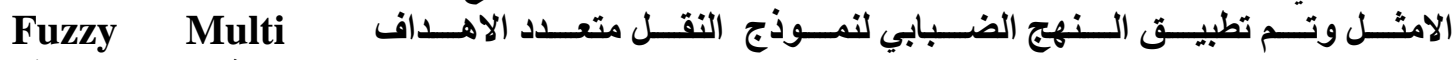
(FMOTP)Objective) Transportation Problem

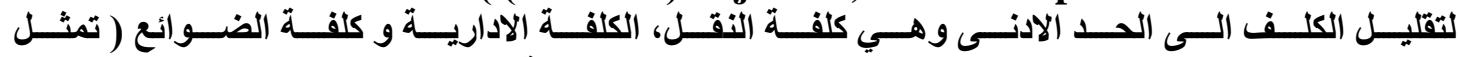

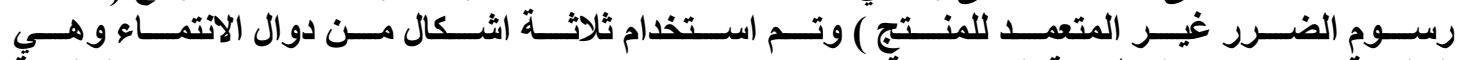

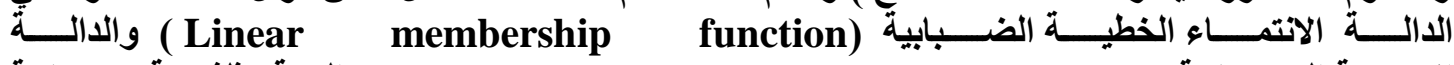

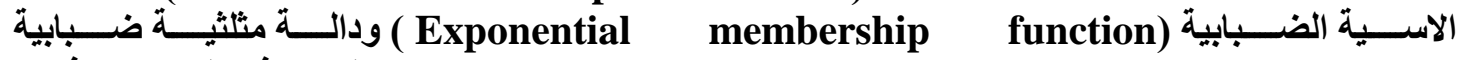

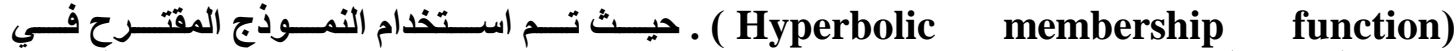

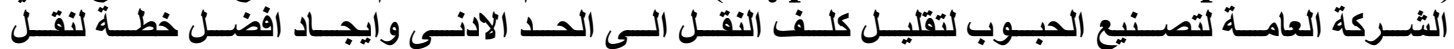
المنتوج وفق القيود المفروضة على النموذج النماني

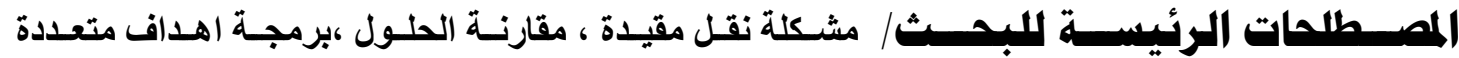
ضبابية ، قيود مختلطة.

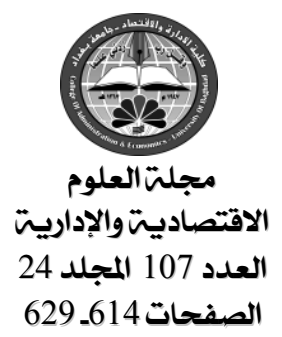




\section{النقلا الضبابي المقيد متعرد الاهدافـ مع قيور مختلطة باستخدام נوال اتتهاء مختلفة}

\section{(1) (1) (القدهمة}

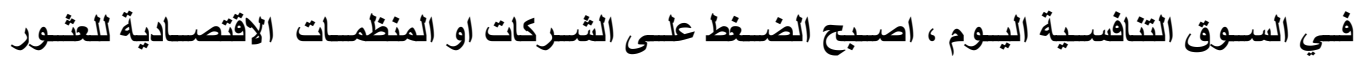

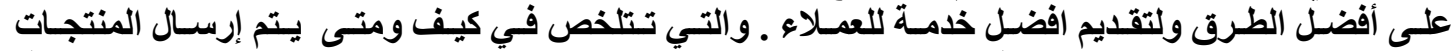

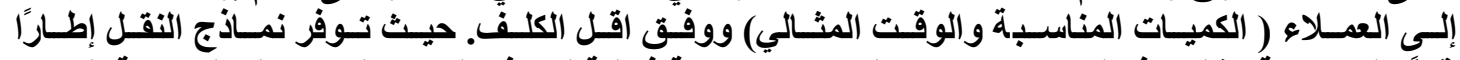

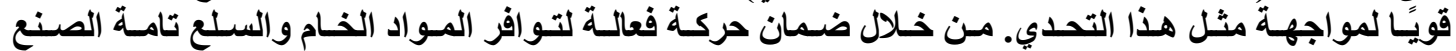

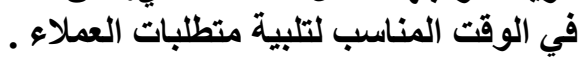

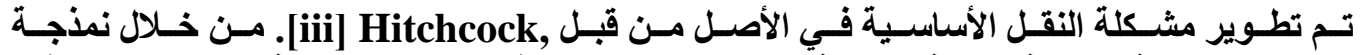

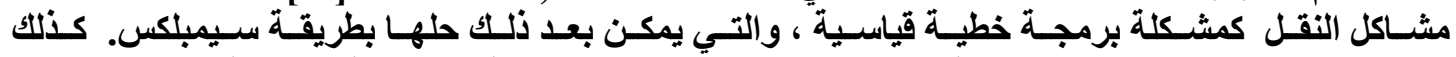

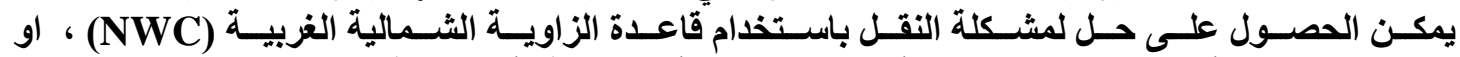

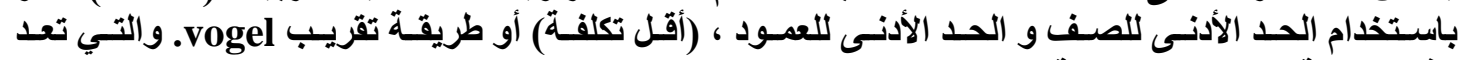

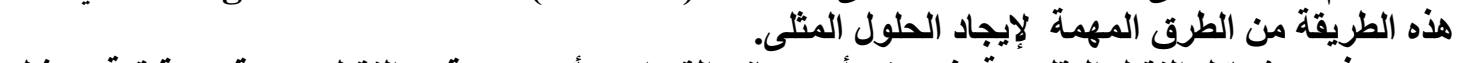

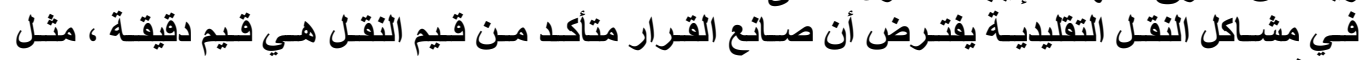
التكلفة وتوافر العرض او طلب المن المنتج.

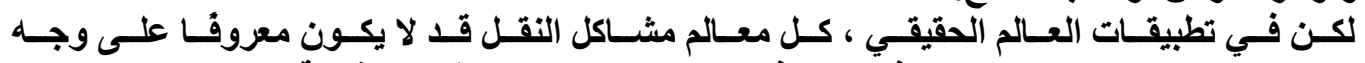

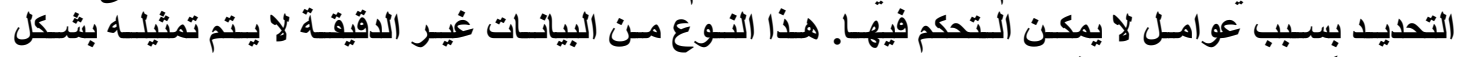

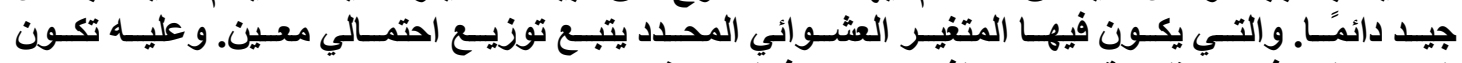

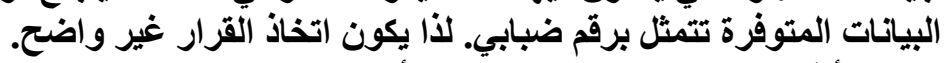

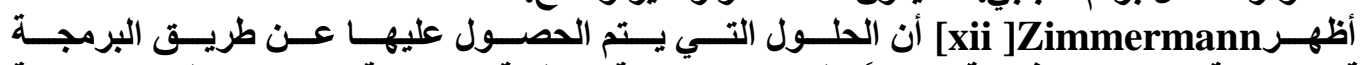

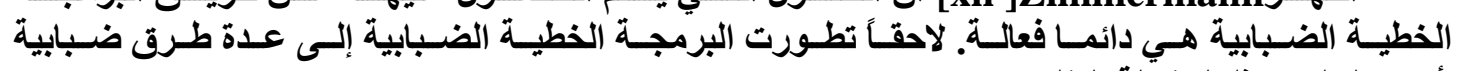

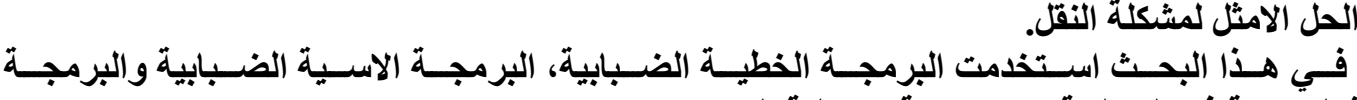
لأيجاد الحل الامثل لمشكلة النقل.

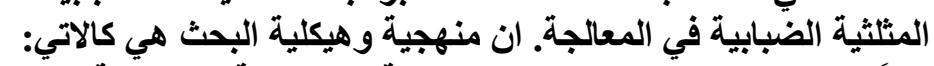

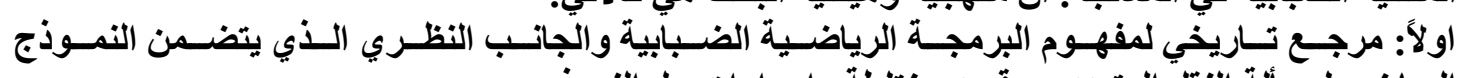

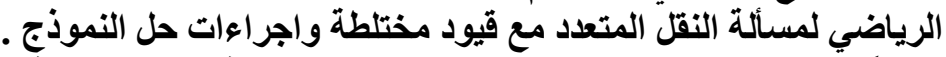

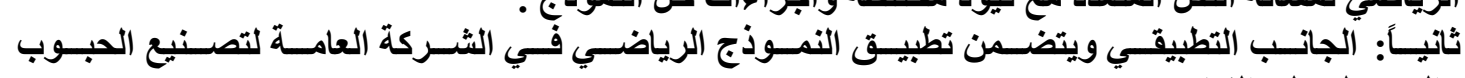

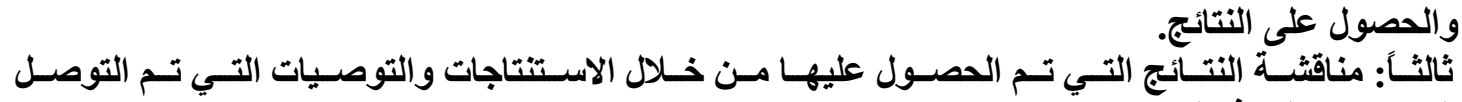
اليها من خلا: منافل هذا البحث.

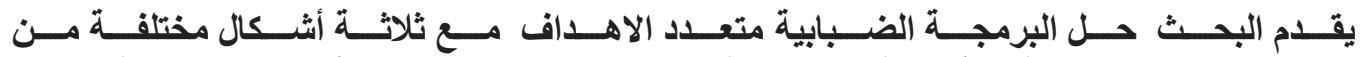

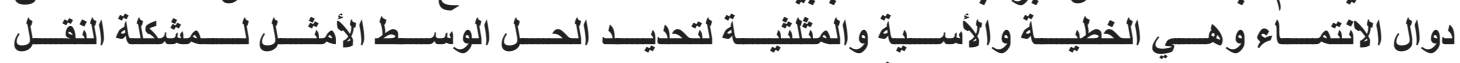

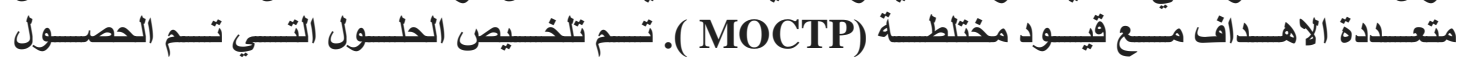

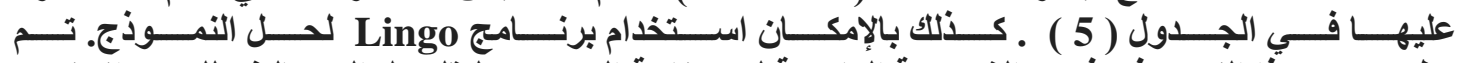

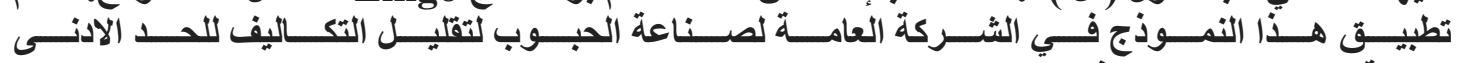
للشركة من خلال بناء انموذج ريّاضي متعدد الاهداف. 


\section{النقلا الفبابيا المقيد متعدر الاهدافـ مم قيور مختلطة باستخدام} נوال اتتماء مختلفة

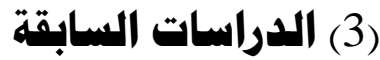

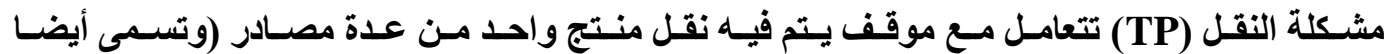

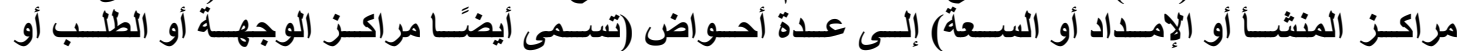

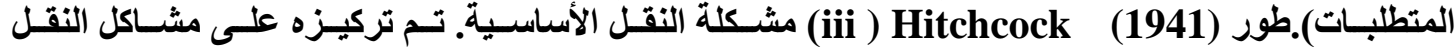

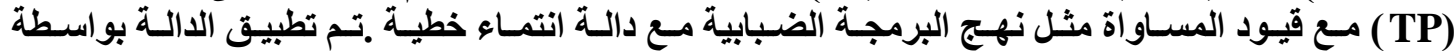

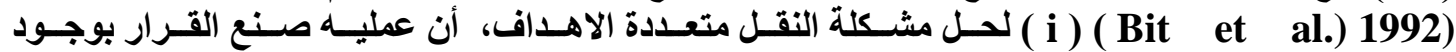

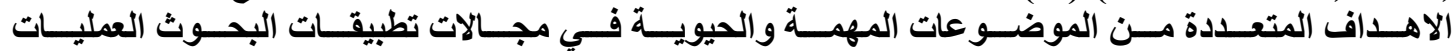

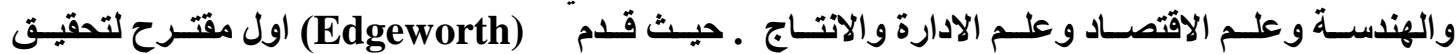

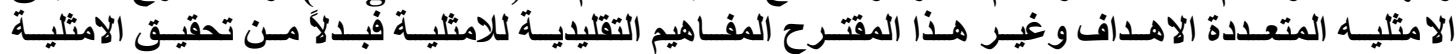

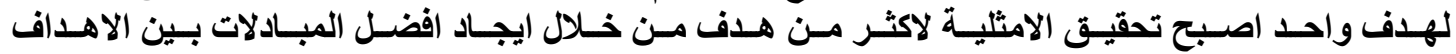

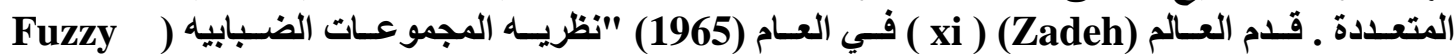

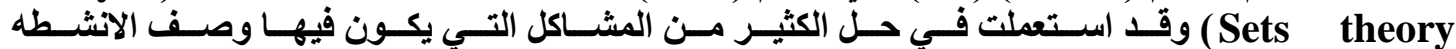

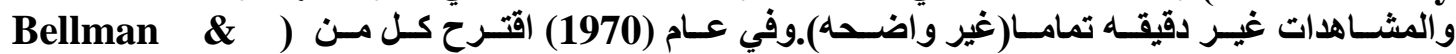

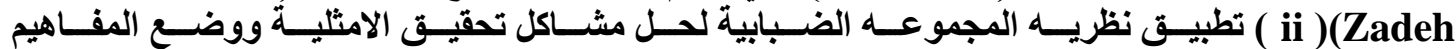

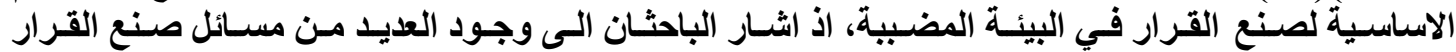

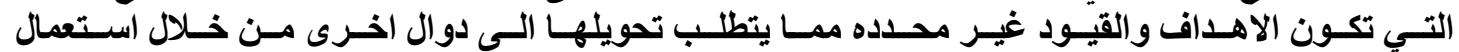

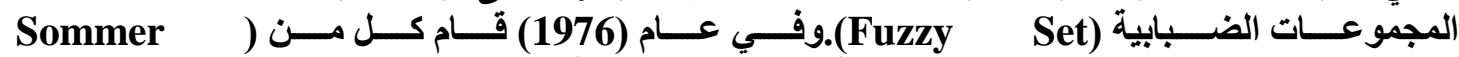

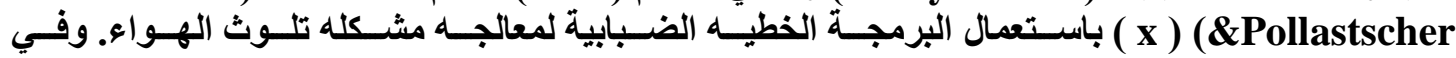

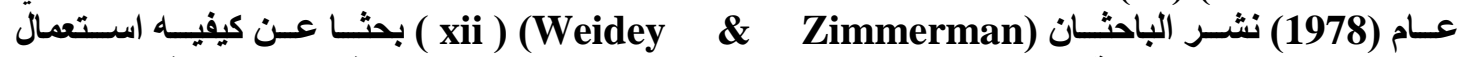

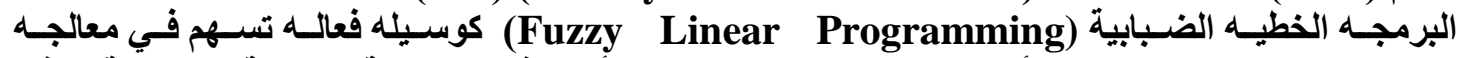

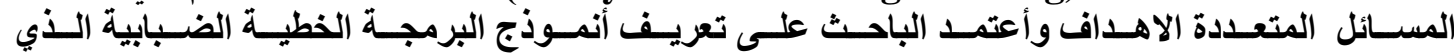

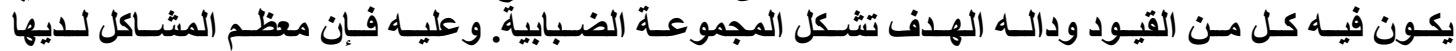

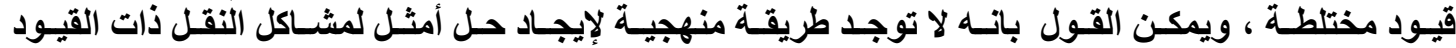
المختلطة.

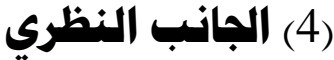

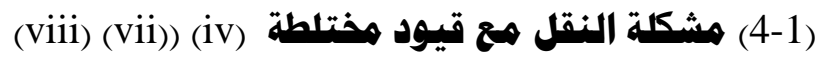

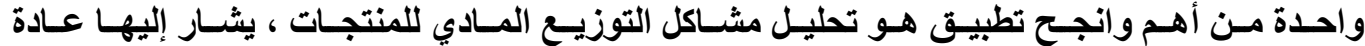

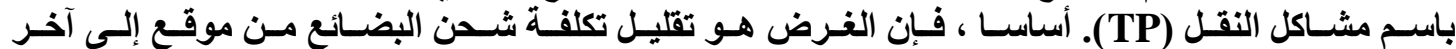

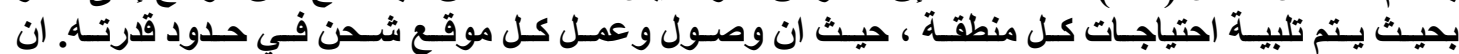

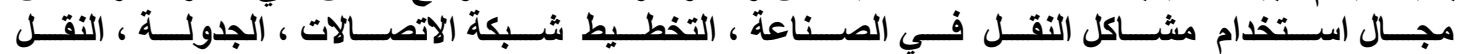

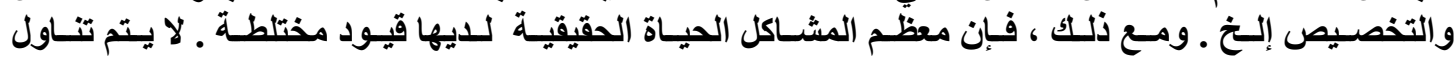

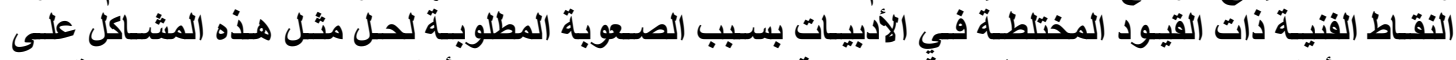

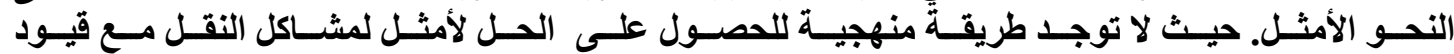
مختلطة.

(viii) (vii) (iv) (4-2) الصيغة الرياضية للنهموذجة (4) لنفتــرض $m$ المصـــــــ ، لـتكن

$$
\text { . }(j=1,2, \ldots, n)
$$




\section{النقل الضبابي المقيد متعدر الاهدافـ مع قيور مختلطة باستخدام} נوال اتتهاء مختلفة

ان صيغة النموذج الرياضي لمشكلة النقل متعددة الاهداف مع قيود مختلطة هي كالاتي:

$$
\left.\begin{array}{c}
\operatorname{Min} Z^{k}=\sum_{i=1}^{m} \sum_{j=1}^{n} c_{i j}^{k} x_{i j}, k=1,2, \ldots, K \\
\text { Subject to } \sum_{j=1}^{n} x_{i j}\{\leq /=/ \geq\} a_{i}, i=1,2, \ldots, m \\
\sum_{i=1}^{m} x_{i j}\{\leq /=/ \geq\} b_{j}, j=1,2, \ldots, n \\
0 \leq x_{i j} \leq r_{i j}
\end{array}\right\}
$$

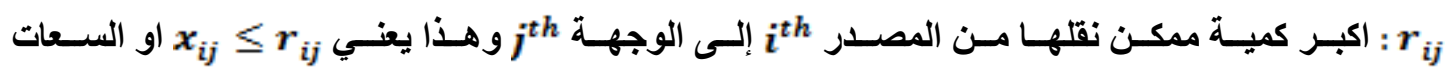

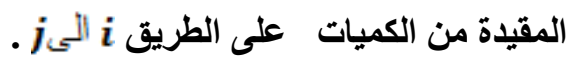

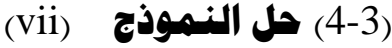

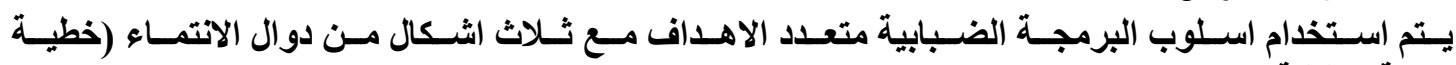
(1) اسية ،مثثية ) وهي كالاتي :-

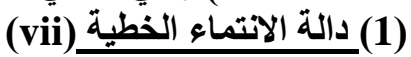
لكل دالة هلف ضبابية خطية تعرف دالة الانية الانتماء

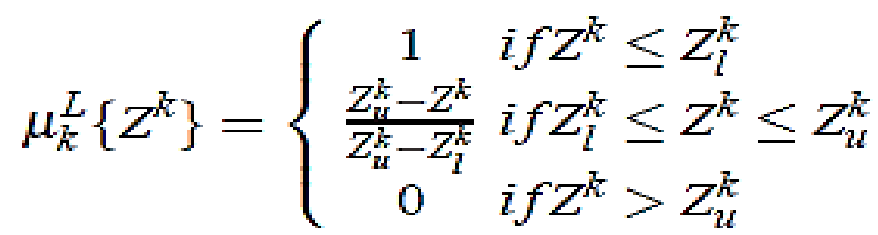

عنـدما

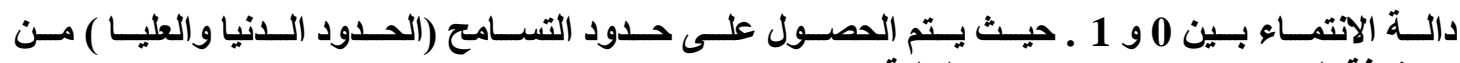
مصفوفة العائد (payoff matrix) مائل التالية :

$$
\text { PayoffMatrix }=\left[\begin{array}{ccccc} 
& Z^{1} & Z^{2} & \ldots & Z^{k} \\
x_{i j}^{(1)} & Z^{\mathbf{l}}\left(x_{i j}^{(1)}\right) & Z^{2}\left(x_{i j}^{(1)}\right) & \cdots & Z^{k}\left(x_{i j}^{(1)}\right) \\
x_{i j}^{(2)} & Z^{\mathbf{l}}\left(x_{i j}^{(2)}\right) & Z^{2}\left(x_{i j}^{(2)}\right) & \cdots & Z^{k}\left(x_{i j}^{(2)}\right) \\
\vdots & \vdots & \vdots & \vdots & \vdots \\
x_{i j}^{(k)} & Z^{1}\left(x_{i j}^{(k)}\right) & Z^{2}\left(x_{i j}^{(k)}\right) & \cdots & Z^{k}\left(x_{i j}^{(k)}\right)
\end{array}\right] ; i=1,2, \ldots, m ; j=1,2, \ldots, n
$$

حيـث

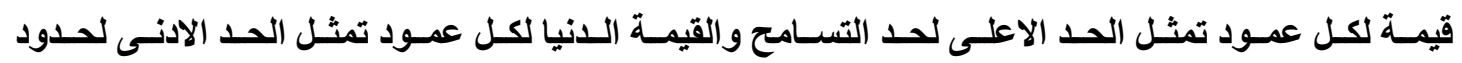

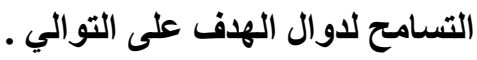




\section{النقل الضبابيا المقيد متعدر الاهدافـ مم قيور مختلطة باستخدام נوال اتتماء مختلفة}

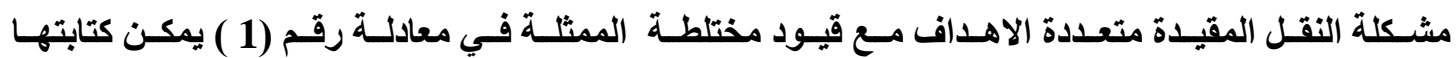
Minimize $\lambda$

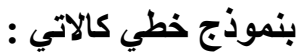

$$
\begin{array}{ll}
\text { Subject to } & \frac{z_{v}^{\mathbf{k}}-Z^{\mathbf{k}}}{z_{\mathbf{u}}^{k}-Z_{i}^{k}} \geq \lambda \\
& \sum_{j=1}^{n} x_{i j}\{\leq /=/ \geq\} a_{i}, i=1,2, \ldots, m \\
& \sum_{i=1}^{m} x_{i j}\{\leq /=/ \geq\} b_{j}, j=1,2, \ldots, n \\
& \lambda \geq 0 \\
& 0 \leq x_{i j} \leq r_{i j}
\end{array}
$$

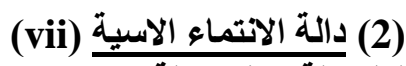
$\mu_{k}^{E}\left(z^{k}\right)$ لكل دالة هدف دالة الانتماء الأبية (2) الأسية

$$
\mu_{k}^{E}\left\{Z^{k}\right\}=\left\{\begin{array}{cl}
\frac{1}{\exp \left(\frac{-\alpha\left(Z^{k}-Z_{j}^{k}\right)}{Z_{u}^{k}-Z_{l}^{k}}\right)-\exp (-\alpha)} & \text { if } Z^{k} \leq Z_{l}^{k} \\
\hline 1-\exp (-\alpha) & \text { if } Z_{l}^{k} \leq Z^{k} \leq Z_{u}^{k} \\
0 & \text { if } Z^{k}>Z_{u}^{k} \text { and } \alpha \rightarrow \infty
\end{array}\right.
$$

حيث ان 0 الان الاهدا مع قيود مختلطة يمكن كتابتها بصيغة غير خطية كالاتي:

$$
\left.\begin{array}{ll}
\text { Minimize } \lambda & \\
\text { Subject to } & \frac{\exp \left(\frac{-\alpha\left(Z^{k}-Z_{j}^{k}\right)}{z_{u}^{k}-Z_{j}^{k}}\right)-\exp (-\alpha)}{1-\exp (-\alpha)} \geq \lambda \\
& \sum_{j=1}^{n} x_{i j}\{\leq /=/ \geq\} a_{i}, i=1,2, \ldots, m \\
& \sum_{i=1}^{m} x_{i j}\{\leq /=/ \geq\} b_{j}, j=1,2, \ldots, n \\
& \lambda \geq 0 \\
& 0 \leq x_{i j} \leq r_{i j}
\end{array}\right\}
$$




\section{النقل الفبابيا المقيد متعدر الاهدافا مع قيور مختلطة باستخدام נوال اتتهاء مختلفة}

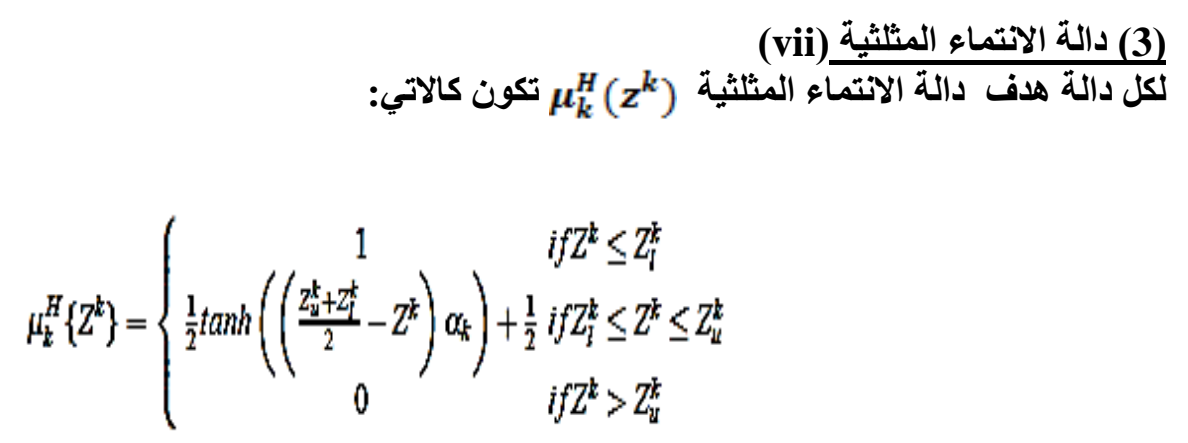

حيـث قدمها Zimmermann, 1985

$$
=\frac{1}{2} \Leftrightarrow Z^{k}=\frac{1}{2}\left(\left(Z_{u}^{k}+Z_{l}^{k}\right) \cdot \mu_{k}^{H}\right.
$$

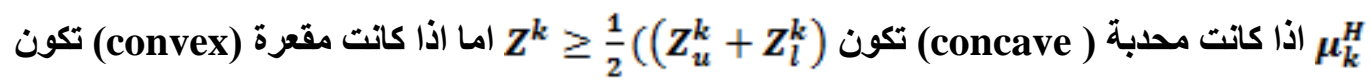

$$
\begin{aligned}
& \text {. } Z^{k} \leq \frac{1}{2}\left(\left(Z_{u}^{k}+Z_{l}^{k}\right)\right.
\end{aligned}
$$

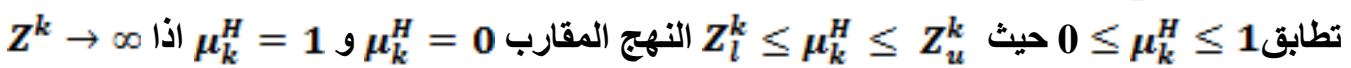

$$
\begin{aligned}
& \text { و }
\end{aligned}
$$

الان مشكلة النقل المقيدة متعددة الاهداف مع قيود مختلطة يمكن كتابتها بصيغة غير خطية كالاتي:

Minimize $\lambda$

Subject to $\frac{1}{2} \tanh \left(\left(\frac{Z_{u}^{k}+Z_{l}^{k}}{2}-Z^{k}\right) \alpha_{k}\right)+\frac{1}{2} \geq \lambda$

$$
\left.\begin{array}{l}
\sum_{j=1}^{n} x_{i j}\{\leq /=/ \geq\} a_{i}, i=1,2, \ldots, m \\
\sum_{i=1}^{m} x_{i j}\{\leq /=/ \geq\} b_{j}, j=1,2, \ldots, n \\
\lambda \geq 0 \\
0 \leq x_{i j} \leq r_{i j}
\end{array}\right\}
$$

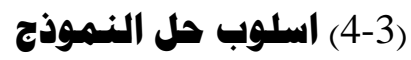

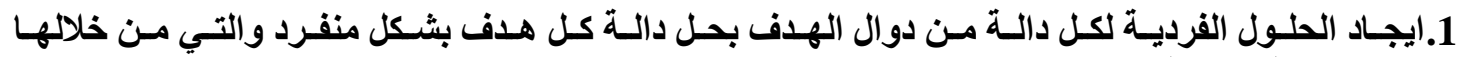

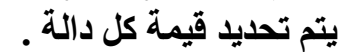

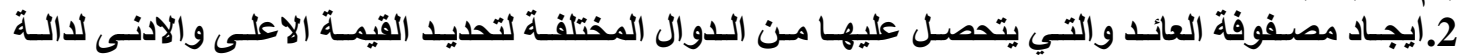




\section{النقل الضبابيا المقيد متعدر الاهدافـ مم قيور مختلطة باستخدام}

دوالا اتتماء مختلفة

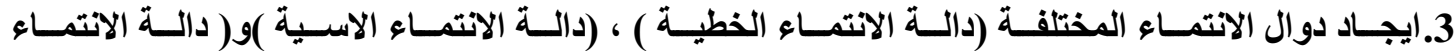

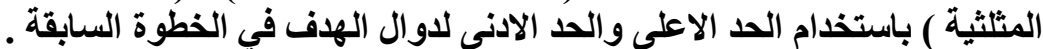

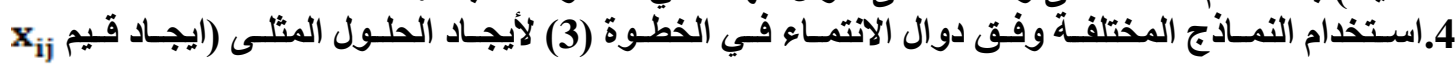

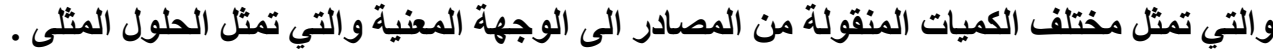

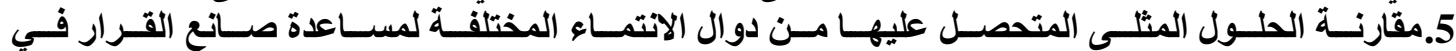

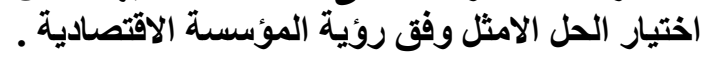

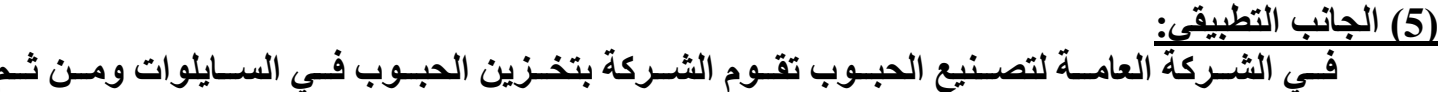

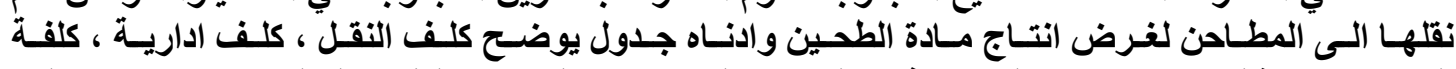

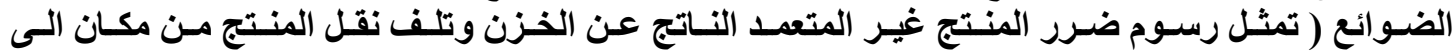

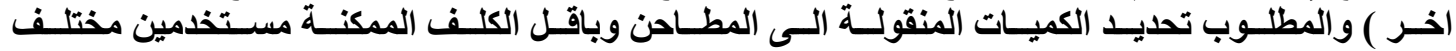
النماذج الضبابية .

جلول رقم (1) يمثل كلف النقل (الف دينار)

\begin{tabular}{|c|c|c|c|c|c|c|}
\hline المطاحن & بغداد ب بغاد & النصر & الجلبي & العطيفية & الخنساء & العرض \\
\hline التاجي & 20 & 12 & 2 & 7 & 16 & $\leq 15360$ \\
\hline الرصافة & 3 & 6 & 20 & 22 & 22 & $\leq 35110$ \\
\hline الدورة & 10 & 5 & 10 & 7 & 11 & $\leq 17184$ \\
\hline خان ضاري & 21 & 17 & 15 & 8 & 3 & $\leq 48000$ \\
\hline خان بني سعد & 15 & 16 & 20 & 11 & 21 & $\leq 16184$ \\
\hline الطب & $\geq 2066$ & $\geq 2859$ & $\geq 3100$ & $\geq 1959$ & $\geq 1733$ & \\
\hline
\end{tabular}
جدول رقم (2) يمثل الكلف الادارية (الف دينار)

\begin{tabular}{|c|c|c|c|c|c|c|}
\hline المطاحن & بغذاد ب بذاد & النصر & الجلبي & العطيفية & الخنساء & العرض \\
\hline التاجي & 12 & 12 & 12 & 12 & 12 & $\leq 15360$ \\
\hline الرصافة & 4 & 4 & 4 & 4 & 4 & $\leq 35110$ \\
\hline الدورة & 10 & 10 & 10 & 10 & 10 & $\leq 17184$ \\
\hline خان ضاري & 3 & 3 & 3 & 3 & 3 & $\leq 48000$ \\
\hline خان بني سعد & 6 & 6 & 6 & 6 & 6 & $\leq 16184$ \\
\hline الطلب & $\geq 2066$ & $\geq 2859$ & $\geq 3100$ & $\geq 1959$ & $\geq 1733$ & \\
\hline
\end{tabular}




\section{النقل الضبابي المقيد متعرد الاهدافـ مع قيود مختلطة باستخدام נوال اتتماء مختلفة}

جدول رقم ( 3) يمثل كلف الضوائع (الف دينار)

\begin{tabular}{|c|c|c|c|c|c|c|}
\hline المطاحن & بغداد بل بل باد & النصر & الجلبي & العطيفية & الخنساء & العرض \\
\hline التاجي & 2 & 1.2 & 0.1 & 0.5 & 1.5 & $\leq 15360$ \\
\hline الرصافة & 0.5 & 0.75 & 1.4 & 1.2 & 2 & $\leq 35110$ \\
\hline الاورة & 1 & 0.5 & 1.1 & 0.4 & 1 & $\leq 17184$ \\
\hline خان ضاري & 2.5 & 1.5 & 1.25 & 0.85 & 0.2 & $\leq 48000$ \\
\hline خان بني سعد & 2.2 & 1.3 & 1.2 & 0.9 & 1.8 & $\leq 16184$ \\
\hline الطلب & $\geq 2066$ & $\geq 2859$ & $\geq 3100$ & $\geq 1959$ & $\geq 1733$ & \\
\hline
\end{tabular}

القيود المفروضة على الكميات المنقولة (طن) من السايلوات الى المطاحن للجداول (1) و(2) و(3). $36 \leq x_{11} \leq 490,24 \leq x_{12} \leq 662,29 \leq x_{13} \leq 766,29 \leq x_{14} \leq 795$, $21 \leq x_{15} \leq 563,32 \leq x_{21} \leq 328,58 \leq x_{22} \leq 424,36 \leq x_{23} \leq 381$ $23 \leq x_{24} \leq 437,50 \leq x_{25} \leq 318,58 \leq x_{31} \leq 331,45 \leq x_{32} \leq 396$ $15 \leq x_{33} \leq 534,23 \leq x_{34} \leq 249,12 \leq x_{35} \leq 265,23 \leq x_{41} \leq 393$ $29 \leq x_{42} \leq 212,18 \leq x_{43} \leq 370,36 \leq x_{44} \leq 219,47 \leq x_{45} \leq 437$ $11 \leq x_{51} \leq 300,21 \leq x_{52} \leq 343,13 \leq x_{53} \leq 258,21 \leq x_{54} \leq 323$ $12 \leq x_{55} \leq 322$.

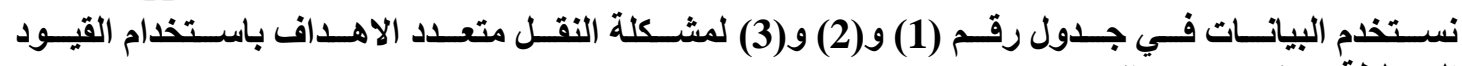
المختلطة حيث ستكون كالاتي:

$\operatorname{Min} Z_{1}=\left(20 x_{11}+12 x_{21}+2 x_{31}+7 x_{41}+16 x_{51}+3 x_{12}+6 x_{22}+20 x_{32}+22 x_{42}+22 x_{52}+10\right.$ $x_{13}+5 x_{23}+10 x_{33}+7 x_{43}+11 x_{53}+21 x_{14}+17 x_{24}+15 x_{34}+8 x_{44}+3 x_{54}+15 x_{15}+16 x_{25}+20$ $\left.x_{35}+11 x_{45}+21 x_{55}\right)$

$\operatorname{MinZ}_{2}=\left(12 x_{11}+12 x_{21}+12 x_{31}+12 x_{41}+12 x_{51}+4 x_{12}+4 x_{22}+4 x_{32}+4 x_{42}+4 x_{52}+10\right.$ $x_{13}+10 x_{23}+10 x_{33}+10 x_{43}+10 x_{53}+3 x_{14}+3 x_{24}+3 x_{34}+3 x_{44}+3 x_{54}+6 x_{15}+6 x_{25}+6 x_{35}+6$ $\left.x_{45}+6 x_{55}\right)$

$\operatorname{Min} Z_{3}=\left(2 x_{11}+1.2 x_{21}+0.1 x_{31}+0.5 x_{41}+1.5 x_{51}+0.5 x_{12}+0.75 x_{22}+1.4 x_{32}+1.2 x_{42}+2\right.$ $x_{52}+x_{13}+0.5 x_{23}+1.1 x_{33}+0.4 x_{43}+x_{53}+2.5 x_{14}+1.5 x_{24}+1.25 x_{34}+0.85 x_{44}+0.2 x_{54}+2.2$ $\left.x_{15}+1.3 x_{25}+1.2 x_{35}+0.9 x_{45}+1.8 x_{55}\right)$

S.t.

قيود العرض والطلب للمنتج

$\sum_{j}^{5} x_{1 j} \leq 15360, \sum_{j}^{5} x_{2 j} \leq 35110, \sum_{j}^{5} x_{3 j} \leq 17184, \sum_{j}^{5} x_{4 j} \leq 48000$

$\sum_{j}^{5} x_{5 j} \leq 16184, \sum_{i}^{5} x_{i 1} \geq 2066, \sum_{i}^{5} x_{i 2} \geq 2859, \sum_{i}^{5} x_{i 3} \geq 3100$ $\sum_{i}^{5} x_{i 4} \geq 1959, \sum_{i}^{5} x_{i 5} \geq 1733$, 


\section{النقل الفبابيا المقيد متعدر الاهدافـ مع قيور مختلطة باستخدام دوال اتتماء مختلفة}

$$
\text { القيود المفروضة على الكميات المنقولة (طن) من السايلوات الى المطاحن }
$$

$36 \leq x_{11} \leq 490,24 \leq x_{12} \leq 662,29 \leq x_{13} \leq 766,29 \leq x_{14} \leq 795$,

$21 \leq x_{15} \leq 563,32 \leq x_{21} \leq 328,58 \leq x_{22} \leq 424,36 \leq x_{23} \leq 381$

$23 \leq x_{24} \leq 437,50 \leq x_{25} \leq 318,58 \leq x_{31} \leq 331,45 \leq x_{32} \leq 396$

$15 \leq x_{33} \leq 534,23 \leq x_{34} \leq 249,12 \leq x_{35} \leq 265,23 \leq x_{41} \leq 393$

$29 \leq x_{42} \leq 212,18 \leq x_{43} \leq 370,36 \leq x_{44} \leq 219,47 \leq x_{45} \leq 437$

$11 \leq x_{51} \leq 300,21 \leq x_{52} \leq 343,13 \leq x_{53} \leq 258,21 \leq x_{54} \leq 323$

$12 \leq x_{55} \leq 322$

يتم الحصول على الحلول المثلى الفردية لكل هدف بحل المثكلة اعلاه بثكل منفصل لكل هدف باستخدام

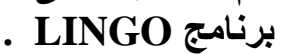

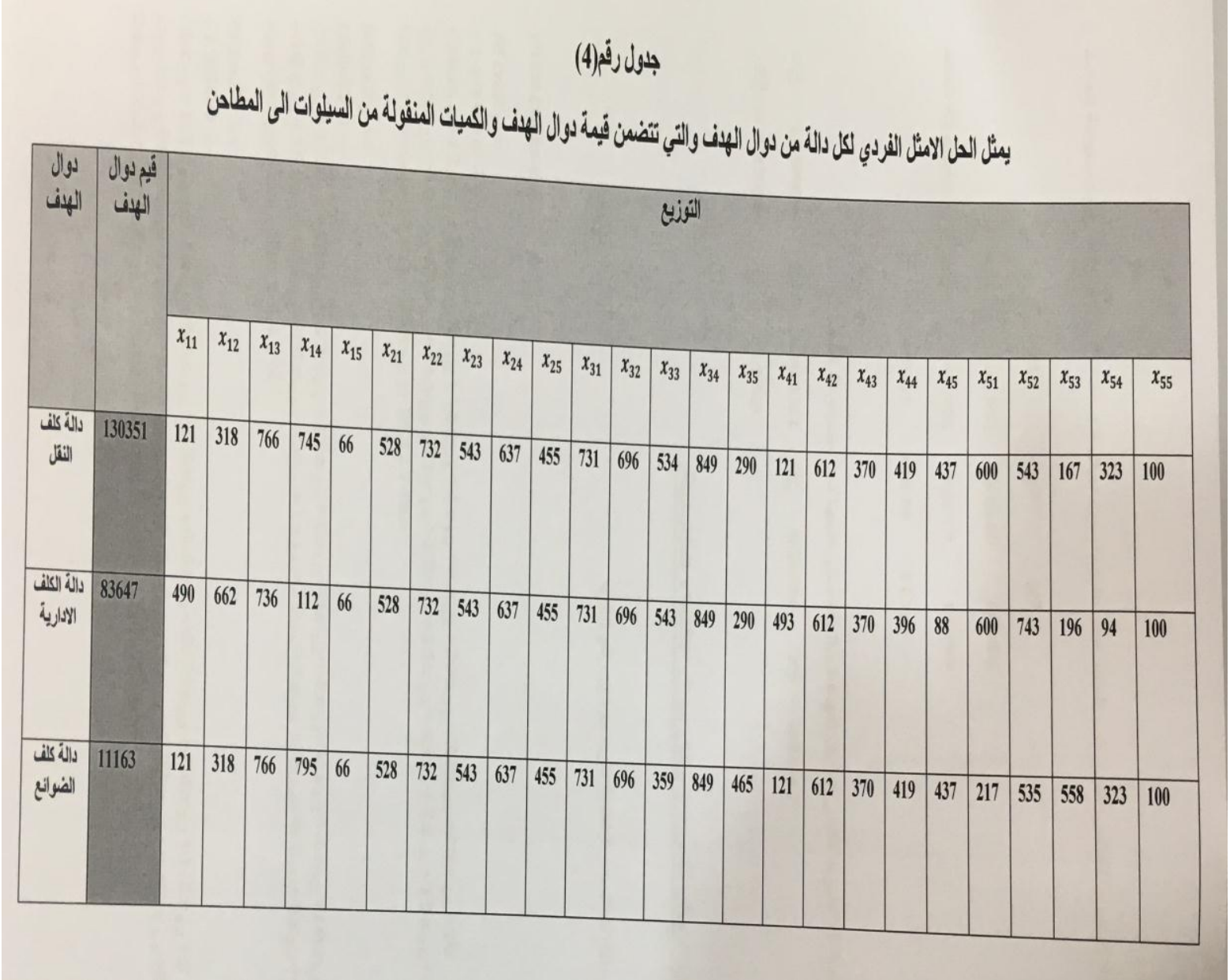




\section{النقلا الفبابي المقيد متعدر الاهدافـ مع قيور مختلطة باستخدام دوالا اتتماء مختلفة}

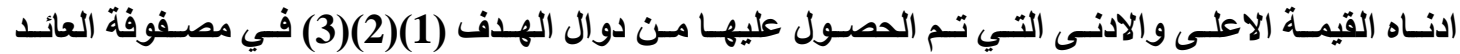
مصفوفة العائد $\left.\begin{array}{cccc} & Z^{1} & Z^{2} & Z^{3} \\ x_{i j}^{1} & 130351 & 84049 & 11539 \\ x_{i j}^{2} & 149906 & 83647 & 13309 \\ x_{i j}^{3} & 143931 & 85676 & 11163\end{array}\right]$ التالية: - n (الناه

يتم تحديد القيمة الاعلى والادنى لاوال الهدف والمتحصل عليها من مصفوفة العائد $Z_{u}^{1}=149906 \quad Z_{l}^{1}=130351 ; Z_{u}^{2}=85676 \quad Z_{l}^{2}=83647$

$$
Z_{u}^{3}=13309 \quad Z_{l}^{3}=11163
$$

وولإيجاد الحل الامثل للنموذج وفق دوال الانتماء الضبابية:

اولًَِ نستخدم دالة الاتثماء الخطية في نموذج رقم (1)

\section{minimize $\lambda$}

$\operatorname{Min} Z_{1}=\left(149906-\left(20 x_{11}+12 x_{21}+2 x_{31}+7 x_{41}+16 x_{51}+3 x_{12}+6 x_{22}+20 x_{32}+22 x_{42}\right.\right.$ $+22 x_{52}+10 x_{13}+5 x_{23}+10 x_{33}+7 x_{43}+11 x_{53}+21 x_{14}+17 x_{24}+15 x_{34}+8 x_{44}+3 x_{54}+15 x_{15}$ $\left.\left.+16 x_{25}+20 x_{35}+11 x_{45}+21 x_{55}\right)\right) \geq 12748 \lambda$.

$\operatorname{MinZ}_{2}=\left(85676-\left(12 x_{11}+12 x_{21}+12 x_{31}+12 x_{41}+12 x_{51}+4 x_{12}+4 x_{22}+4 x_{32}+4 x_{42}+\right.\right.$ $4 x_{52}+10 x_{13}+10 x_{23}+10 x_{33}+10 x_{43}+10 x_{53}+3 x_{14}+3 x_{24}+3 x_{34}+3 x_{44}+3 x_{54}+6 x_{15}+6 x_{25}$ $\left.\left.+6 x_{35}+6 x_{45}+6 x_{55}\right)\right) \geq 6141 \lambda$

$\operatorname{Min} Z_{3}=\left(13309-\left(2 x_{11}+1.2 x_{21}+0.1 x_{31}+0.5 x_{41}+1.5 x_{51}+0.5 x_{12}+0.75 x_{22}+1.4 x_{32}\right.\right.$ $+1.2 x_{42}+2 x_{52}+x_{13}+0.5 x_{23}+1.1 x_{33}+0.4 x_{43}+x_{53}+2.5 x_{14}+1.5 x_{24}+1.25 x_{34}+0.85 x_{44}+$ $\left.\left.0.2 x_{54}+2.2 x_{15}+1.3 x_{25}+1.2 x_{35}+0.9 x_{45}+1.8 x_{55}\right)\right) \geq 2146 \lambda$

S.t.

قيود العرض والطلب للمنتج

$\sum_{j}^{5} x_{1 j} \leq 15360, \sum_{j}^{5} x_{2 j} \leq 35110, \sum_{j}^{5} x_{3 j} \leq 17184, \sum_{j}^{5} x_{4 j} \leq 48000$

$\sum_{j}^{5} x_{5 j} \leq 16184, \sum_{i}^{5} x_{i 1} \geq 2066, \sum_{i}^{5} x_{i 2} \geq 2859, \sum_{i}^{5} x_{i 3} \geq 3100$

$\sum_{i}^{5} x_{i 4} \geq 1959, \sum_{i}^{5} x_{i 5} \geq 1733$, 
النقلا الفبابي المقيد متعدر الاهدافا مع قيور مختلطة باستخدام נوال اتتهاء مختلفة

القيود المفروضة على الكميات المنقولة (طن) من السايلوات الى المطاحن

$36 \leq x_{11} \leq 490,24 \leq x_{12} \leq 662,29 \leq x_{13} \leq 766,29 \leq x_{14} \leq 795$,

$21 \leq x_{15} \leq 563,32 \leq x_{21} \leq 328,58 \leq x_{22} \leq 424,36 \leq x_{23} \leq 381$

$23 \leq x_{24} \leq 437,50 \leq x_{25} \leq \mathbf{3 1 8}, 58 \leq x_{31} \leq 331,45 \leq x_{32} \leq 396$

$15 \leq x_{33} \leq 534,23 \leq x_{34} \leq 249,12 \leq x_{35} \leq 265,23 \leq x_{41} \leq 393$

$29 \leq x_{42} \leq 212,18 \leq x_{43} \leq 370,36 \leq x_{44} \leq 219,47 \leq x_{45} \leq 437$

$11 \leq x_{51} \leq 300,21 \leq x_{52} \leq 343,13 \leq x_{53} \leq 258,21 \leq x_{54} \leq 323$

$12 \leq x_{55} \leq 322$

يتم الحصول على النتائج التالية والتي تمثل الحلول المثلى وفق دالـة الانتمساء الخطية : LINGO باستخدام

برنامج

$x_{11}^{*}=490 ; x_{12}^{*}=85 ; x_{13}^{*}=766 ; x_{14}^{*}=162 ; x_{15}^{*}=563$

$x_{21}^{*}=528 ; x_{22}^{*}=732 ; x_{23}^{*}=543 ; x_{24}^{*}=637 ; x_{25}^{*}=455$

$x_{31}^{*}=556 ; x_{32}^{*}=696 ; x_{33}^{*}=534 ; x_{34}^{*}=849 ; x_{35}^{*}=465$

$x_{41}^{*}=493 ; x_{42}^{*}=543 ; x_{43}^{*}=67 ; x_{44}^{*}=419 ; x_{45}^{*}=437$

$x_{51}^{*}=217 ; x_{52}^{*}=743 ; x_{53}^{*}=350 ; x_{54}^{*}=323 ; x_{55}^{*}=100$

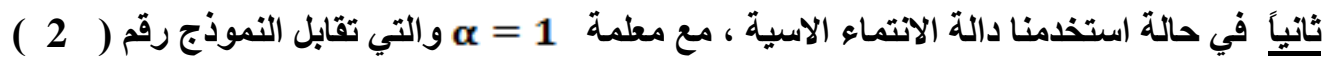
يمكن صياغة النموذج بالثكل التالي:

minimize $\lambda$

$\frac{e^{-\left(z_{1}-149906\right)} e^{-1}}{1-e^{-1}} \geq \lambda$

$\frac{e^{-\left(z_{2}-185676\right)} e^{-1}}{1-e^{-1}} \geq \lambda$

$\frac{e^{-\left(z_{3}-13309\right)} e^{-1}}{1-e^{-1}} \geq \lambda$

S.t.

قيود العرض والطلب

$\sum_{j}^{5} x_{5 j} \leq 16184, \sum_{i}^{5} x_{i 1} \geq 2066, \sum_{i}^{5} x_{i 2} \geq 2859, \sum_{i}^{5} x_{i 3} \geq 3100$

$\sum_{i}^{5} x_{i 4} \geq 1959, \sum_{i}^{5} x_{i 5} \geq 1733$, 
النقلا الفبابي المقيد متعدر الاهدافا مع قيور مختلطة باستخدام נوال اتتهاء مختلفة

القيود المفروضة على الكميات المنقولة (طن) من السايلوات الى المطاحن

$36 \leq x_{11} \leq 490,24 \leq x_{12} \leq 662,29 \leq x_{13} \leq 766,29 \leq x_{14} \leq 795$,

$21 \leq x_{15} \leq 563,32 \leq x_{21} \leq 328,58 \leq x_{22} \leq 424,36 \leq x_{23} \leq 381$

$23 \leq x_{24} \leq 437,50 \leq x_{25} \leq 318,58 \leq x_{31} \leq 331,45 \leq x_{32} \leq 396$

$15 \leq x_{33} \leq 534,23 \leq x_{34} \leq 249,12 \leq x_{35} \leq 265,23 \leq x_{41} \leq 393$

$29 \leq x_{42} \leq 212,18 \leq x_{43} \leq 370,36 \leq x_{44} \leq 219,47 \leq x_{45} \leq 437$

$11 \leq x_{51} \leq 300,21 \leq x_{52} \leq 343,13 \leq x_{53} \leq 258,21 \leq x_{54} \leq 323$

$12 \leq x_{55} \leq 322$

يتم الحصول على النتائج التالية والتي تمثل الحلول المثلى وفق دالة الانتمـاء الاسية : LINGO باستخدام

برنامج

$$
\begin{aligned}
& x_{11}^{*}=490 ; x_{12}^{*}=662 ; x_{13}^{*}=766 ; x_{14}^{*}=795 ; x_{15}^{*}=563 \\
& x_{21}^{*}=528 ; x_{22}^{*}=732 ; x_{23}^{*}=543 ; x_{24}^{*}=518 ; x_{25}^{*}=731 \\
& x_{31}^{*}=731 ; x_{32}^{*}=696 ; x_{33}^{*}=534 ; x_{34}^{*}=849 ; x_{35}^{*}=465 \\
& x_{41}^{*}=493 ; x_{42}^{*}=612 ; x_{43}^{*}=370 ; x_{44}^{*}=419 ; x_{45}^{*}=437 \\
& x_{51}^{*}=600 ; x_{52}^{*}=743 ; x_{53}^{*}=558 ; x_{54}^{*}=323 ; x_{55}^{*}=422
\end{aligned}
$$

minimize $\lambda$

ثالثًاً دالة الانتماء المثلثية في نموذج رقم (3)

$1 / 2 \tanh \left(\left(149906-\left(20 x_{11}+12 x_{21}+2 x_{31}+7 x_{41}+16 x_{51}+3 x_{12}+6 x_{22}+20 x_{32}+22 x_{42}\right.\right.\right.$ $+22 x_{52}+10 x_{13}+5 x_{23}+10 x_{33}+7 x_{43}+11 x_{53}+21 x_{14}+17 x_{24}+15 x_{34}+8 x_{44}+3 x_{54}+15 x_{15}$ $\left.\left.\left.\left.+16 x_{25}+20 x_{35}+11 x_{45}+21 x_{55}\right)\right)\right) 0.0005\right)+0.5 \geq \lambda$

$1 / 2 \tanh \left(\left(85676-\left(12 x_{11}+12 x_{21}+12 x_{31}+12 x_{41}+12 x_{51}+4 x_{12}+4 x_{22}+4 x_{32}+4 x_{42}+4\right.\right.\right.$ $x_{52}+10 x_{13}+10 x_{23}+10 x_{33}+10 x_{43}+10 x_{53}+3 x_{14}+3 x_{24}+3 x_{34}+3 x_{44}+3 x_{54}+6 x_{15}+6 x_{25}+$ $\left.\left.\left.6 x_{35}+6 x_{45}+6 x_{55}\right)\right)(0.001)+0.5\right) \geq \lambda$

$1 / 2 \tanh \left(\left(13309-\left(2 x_{11}+1.2 x_{21}+0.1 x_{31}+0.5 x_{41}+1.5 x_{51}+0.5 x_{12}+0.75 x_{22}+1.4\right.\right.\right.$ $x_{32}+1.2 x_{42}+2 x_{52}+x_{13}+0.5 x_{23}+1.1 x_{33}+0.4 x_{43}+x_{53}+2.5 x_{14}+1.5 x_{24}+1.25 x_{34}+0.85$ $\left.\left.\left.\left.x_{44}+0.2 x_{54}+2.2 x_{15}+1.3 x_{25}+1.2 x_{35}+0.9 x_{45}+1.8 x_{55}\right)\right)\right) 0.003\right)+0.5 \geq \lambda$

S.t.

قيود العرض والطلب

$\sum_{j}^{5} x_{5 j} \leq 16184, \sum_{i}^{5} x_{i 1} \geq 2066, \sum_{i}^{5} x_{i 2} \geq 2859, \sum_{i}^{5} x_{i 3} \geq 3100$

$\sum_{i}^{5} x_{i 4} \geq 1959, \sum_{i}^{5} x_{i 5} \geq 1733$, 


\section{النقل الفبابيا المقيد متعرد الاهداف مع قيور مختلطة باستخدام נوال اتتهاء مختلفة}

$$
\text { القيود المفروضة على الكميات المنقولة (طن) من السايلوات الى المطاحن }
$$

$36 \leq x_{11} \leq 490,24 \leq x_{12} \leq 662,29 \leq x_{13} \leq 766,29 \leq x_{14} \leq 795$,

$21 \leq x_{15} \leq 563,32 \leq x_{21} \leq 328,58 \leq x_{22} \leq 424,36 \leq x_{23} \leq 381$

$23 \leq x_{24} \leq 437,50 \leq x_{25} \leq 318,58 \leq x_{31} \leq 331,45 \leq x_{32} \leq 396$

$15 \leq x_{33} \leq 534,23 \leq x_{34} \leq 249,12 \leq x_{35} \leq 265,23 \leq x_{41} \leq 393$

$29 \leq x_{42} \leq 212,18 \leq x_{43} \leq 370,36 \leq x_{44} \leq 219,47 \leq x_{45} \leq 437$

$11 \leq x_{51} \leq 300,21 \leq x_{52} \leq 343,13 \leq x_{53} \leq 258,21 \leq x_{54} \leq 323$

$12 \leq x_{55} \leq 322$

يتم الحصول على النتائج التالية والتي تمثل الحلول المثلى التي تم الحصول عليها : LINGO باستخدام

$x_{11}^{*}=472 ; x_{12}^{*}=662 ; x_{13}^{*}=67 ; x_{14}^{*}=746 ; x_{15}^{*}=156$

برنامج

$x_{21}^{*}=528 ; x_{22}^{*}=732 ; x_{23}^{*}=539 ; x_{24}^{*}=637 ; x_{25}^{*}=517$

$x_{31}^{*}=731 ; x_{32}^{*}=696 ; x_{33}^{*}=534 ; x_{34}^{*}=849 ; x_{35}^{*}=290$

$x_{41}^{*}=368 ; x_{42}^{*}=612 ; x_{43}^{*}=287 ; x_{44}^{*}=299 ; x_{45}^{*}=391$

$x_{51}^{*}=600 ; x_{52}^{*}=179 ; x_{53}^{*}=558 ; x_{54}^{*}=195 ; x_{55}^{*}=201$

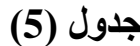

يوضح مقارنة مختلف الحلول المثلى لقيم دوال الهُف

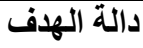

\begin{tabular}{|c|c|c|c|}
\hline \multicolumn{3}{|c|}{ دالة الهـف } & \multirow{2}{*}{ الطريقة } \\
\hline دالة كلف الضوائع & دالة الكلف الادارية & دالة كلف النقل & \\
\hline 12536 & 80369 & 151010 & الدالة الاتتماءة الضبابية \\
\hline 16283 & 105109 & 185480 & الدالة الانتماءة الضبابية \\
\hline 13318 & 82549 & 155074 & الدالة الانتماءة الضبابية \\
\hline
\end{tabular}




\section{النقل الفبابي المقيد متعرد الاهدافـ مع قيود مختلطة باستخدام دوالا اتتماء مختلفة}

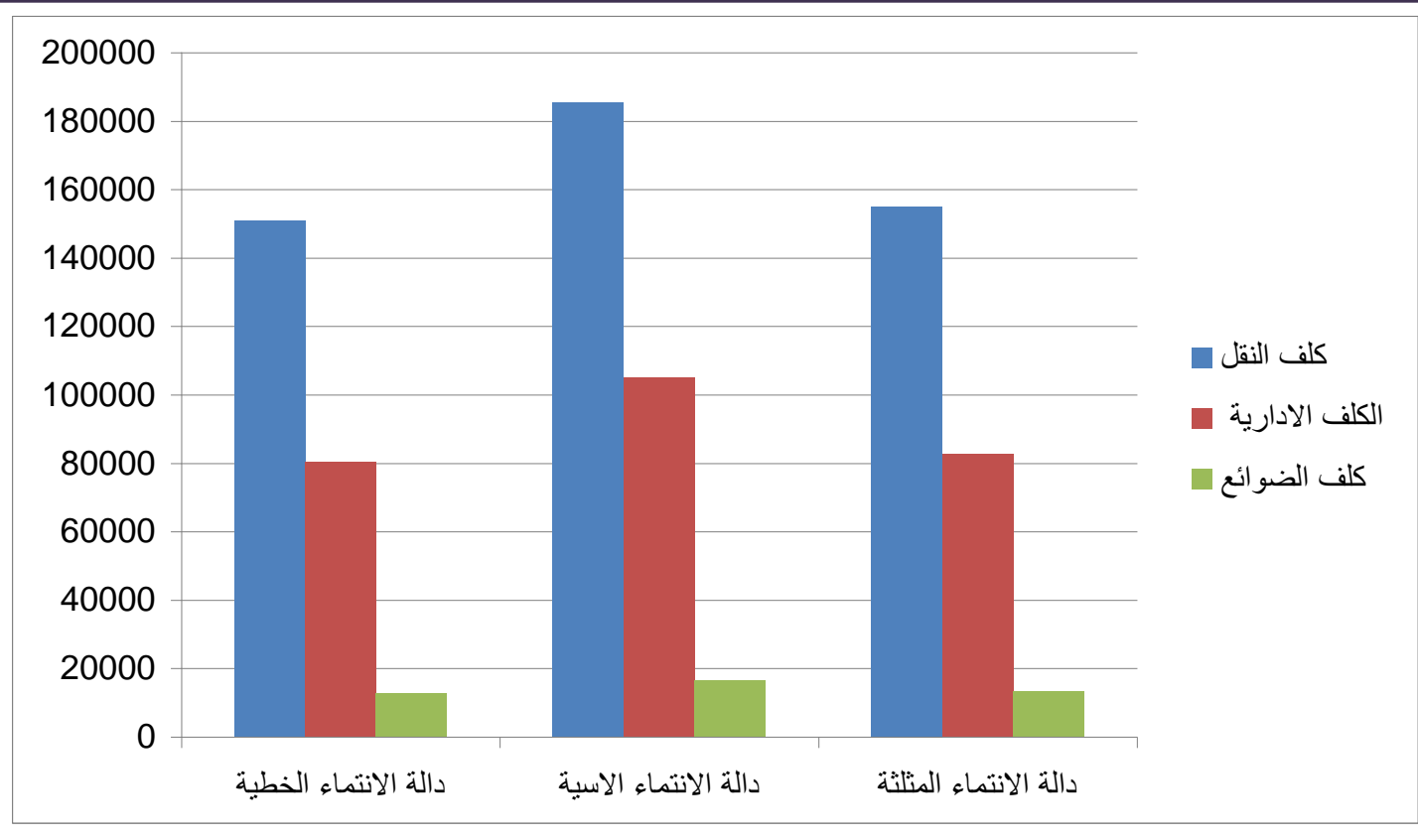

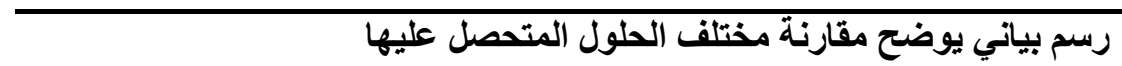

(6) اللذص والاستنتاجات

1 ـ عرض البحث ثلاث انواع من البرمجة الضبابية مما جعل هناك مرونة في رؤية المشكلة بثكل اوسع.

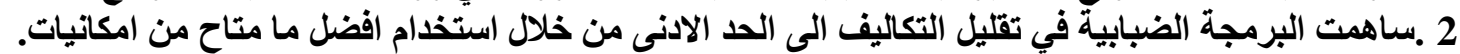

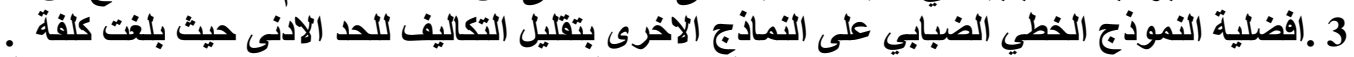

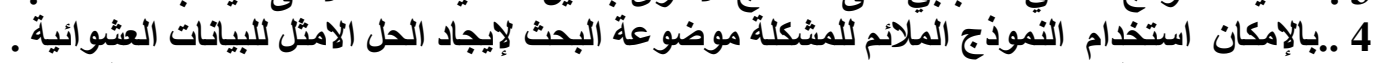
6 ـ تسطيع الثركة تعديل خطظ النقل وفق النتائج المتحصل عليها للحصول على القل كلف ممكنة .

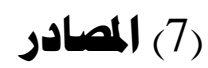

i. Bit, A.K., Biswal, M.P. and Alam, S.S. (1992). Fuzzy programming approach to multi criteria decision making transportation problem, Fuzzy Sets and Systems, 50, 135-141.

ii. Bellman ,R.E.\& Zadeh L.A, Decision making in afuzzy environment, (1970), .management science, vol.17, pp.141-146.

iii. Hitchcock, F.L. (1941). The distribution of a product from several sources to numerous localities, J. Math. Phys., 20, 224-230.

iv. Gupta, N., Ali, I., Bari, A. (2013). A compromise solution for multi-objective chance constraint capacitated transportation.

v. LINGO-User's Guide (2001). "LINGO-User's Guide". Published by LINDO SYSTEMINC., 1415, North Dayton Street, Chicago.

vi. Mondal, R.N., and Hossain, R. (2012). Solving Transportation Problem with Mixed Constraints, Proceedings of the 2012 International Conference on Industrial Engineering and Operations Management Istanbul, Turkey, July 36. 


\section{النقل الفبابيا المقيد متعدر الاهدافـ مع قيور مختلطة باستخدام נوال اتتماء مختلفة}

vii. Neha Gupta_ and Abdul Bari.(2014). Fuzzy Multi-Objective Capacitated Transportation Problem with Mixed Constraints. Journal of Statistics Applications \& Probability. No. 2, 201-209.

viii. Radindra Nath Mondal and Md. Rezwan Hossain. ( 2012). Solving Transportation Problem with Mixed Constraints. International Conference on Industrial Engineering and Operations Management Istanbul, Turkey, July 3 6, 2012

ix. Sakawa, M., Nishizaki, I. and Katagiri, H. (2011). Fuzzy Stochastic Multi objective Programming, Springer.

x. Sommer, G \& Pollatschek, M.A.,(1976) " A Fuzzy Programming Approach to an Air Pollution Regulation Problem" Working Pap, No. 76. Inst.Wirtschaftswiss. R.W.T.H.,Aachen. Systems, 6, 105-228.

xi. Zadeh, 1. A. (1965). Fuzzy sets, Information and Control, 8, 338-353.

xii. Zimmermann, H.-J. (1978). Fuzzy programming and linear programming with several objective functions, Fuzzy Sets and Systems,1, 45-55. 


\section{النقل الفبابيا المقيد متعدر الاهدافـ مع قيور مختلطة باستخدام دوال اتتماء مختلفة}

\section{Fuzzy Multi-Objective Capacitated Transportation Problem with Mixed Constraints using different forms of membership functions}

\section{Abstract}

In this research, the problem of multi- objective modal transport was formulated with mixed constraints to find the optimal solution. The foggy approach of the Multi-objective Transfer Model (MOTP) was applied. There are three objectives to reduce costs to the minimum cost of transportation, administrative cost and cost of the goods. The linear membership function, the Exponential membership function, and the Hyperbolic membership function. Where the proposed model was used in the General Company for the manufacture of grain to reduce the cost of transport to the minimum and to find the best plan to transfer the product according to the restrictions imposed on the model.

Keywords: Capacitated Transportation problem; Compromise Solution; Mixed constraints; Multi-Objective programming. 